\title{
Diphtheria and the respiratory system: Lessons from the 2015 outbreak
}

M Annamalai, MB BCh, DCH (SA), Dip HIV Man (SA), Dip Allerg (SA), FCPaed (SA), Cert Pulm (SA) Paeds

Department of Paediatrics, Stanger Regional Hospital, Stanger, South Africa

Corresponding author: M Annamalai (medeshni@yahoo.com)

South Africa experienced a diphtheria outbreak in KwaZulu-Natal Province between March and August 2015. Diphtheria is a contagious and potentially life-threatening bacterial disease caused by the toxigenic strains of Corynebacterium diphtheriae.

S Afr Respir J 2016;22(2):38-42. DOI:10.7196/SARJ.2016.v22i2.75

\section{Outline of the 2015 Kwa-Zulu Natal Province diphtheria outbreak}

In March 2015, the National Institute of Communicable Diseases (NICD) in South Africa (SA) received a case report of an 8-year-old boy confirmed to have Corynebacterium diphtheriae pharyngitis at Inkosi Albert Luthuli Central Hospital (IALCH), Durban, SA. ${ }^{[1]}$

The patient presented with an acute severe illness, with a massively swollen neck, marked drooling and in respiratory distress. A whitish membrane was noted covering the uvula. The patient required an emergency tracheostomy and was transferred to the paediatric intensive care unit. The patient was treated with penicillin, gentamycin and metronidazole. Diphtheria antitoxin (DAT) was not indicated at the time of presentation. The parent confirmed that the child had received all the diphtheria-containing vaccines until 18 months of age but had missed the 6-year booster vaccine. The National Health Laboratory Services laboratory at IALCH isolated C. diphtheriae from his clinical samples and the isolate was positive for the toxin production. ${ }^{[1]}$ The patient unfortunately died on 22 March 2015. ${ }^{[1]}$

According to the NICD, by July 2015 there were four reported deaths from diphtheria.

In May 2015, the Japanese Ministry of Health, Labour and Welfare organised the nationwide collection and donation of 416 vials of the freeze-dried diphtheria equine antitoxin 'Kaketsuken' (with an approximate value of ZAR2 million). Japan is one of only three countries globally that currently manufactures the DAT.

By June 2015, there were 15 reported cases. Of these, 10 were confirmed cases, 2 were probable and 3 were possible, with no further cases under investigation ${ }^{[2]}$ (refer to case definition below). The cases were reported from two of the 11 districts in KwaZulu-Natal (KZN) - Ugu and Ethekwini (Fig. 1). The age of the cases ranged from 4 to 41 years old (median 10 years). Children under 15 years of age accounted for the majority $(73 \%(11 / 15))$. Of these, $40 \%$ were aged between 5 and 9 years $(6 / 15)$ and males accounted for $60 \%(9 / 15)$.

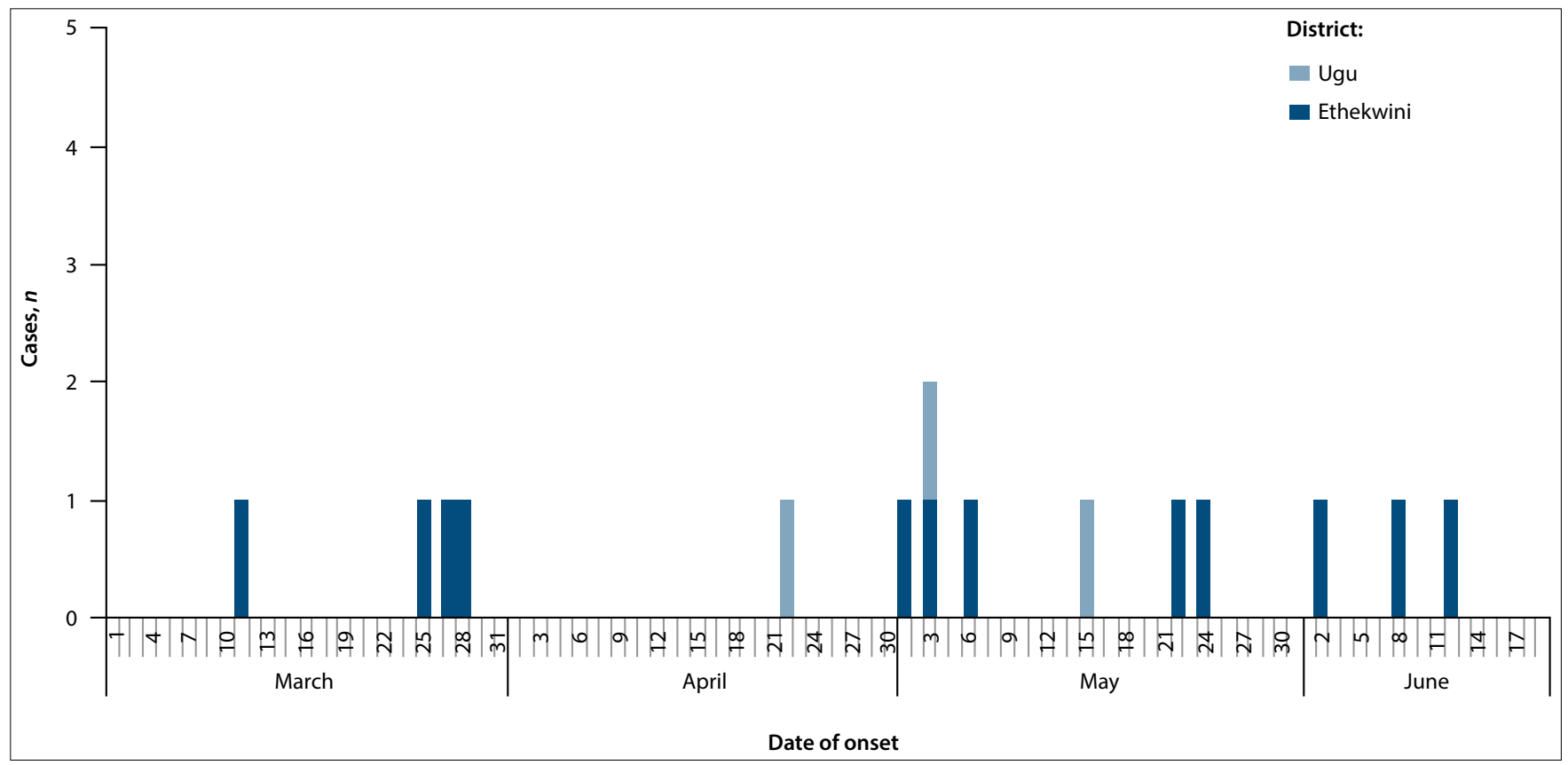

Fig. 1. Epidemic curve illustrating the number of diphtheria cases by date of illness onset and district, KZN, March - 19 June $2015{ }^{[2]}$ 
In the under-22 years age group $(n=13)$, the vaccination history was known for 35\% (5/13) of cases. Of these, only one with probable diphtheria had received all age-appropriate diphtheria-containing vaccine doses. ${ }^{[2]}$

There were three asymptomatic carriers of laboratory-confirmed toxigenic C. diphtheriae. Two of these were epidemiologically linked to two confined areas in Margate (siblings from the same family). The other carrier was epidemiologically linked to a possible case from Umlazi. $^{[2]}$

In August 2015, the NICD released the molecular epidemiology results of the C. diphtheriae outbreak isolates and performed molecular sequencing on isolates from the outbreak. Two novel sequence types were identified, neither of which were related to any other sequence type listed in the global database. ${ }^{[3]}$ Seventeen toxin-producing isolates from the cases and contacts had the same sequence type (ST-378). A second cluster comprised the four non-toxigenic KZN isolates and one of a group of historical non-toxigenic clinical isolates from 1980. These five isolates were of the same sequence type (ST-395). ${ }^{[3]}$

The NICD concluded that it was not yet possible to determine the origin of these outbreak strains, as there were no data describing circulating genotypes in SA. ${ }^{[3]}$

\section{Pathophysiology of diphtheria}

C. diphtheriae is a non-sporulating, Gram-positive bacillus. The name is derived from korynee, meaning 'club' - referring to its clubbed ends. Diphtheria describes the 'leather-hide' characteristic leaky pharyngeal membrane that is formed. ${ }^{[4]}$

The species is subdivided into four biovars - gravis, intermedius, mitis and belfanti. Currently there are 86 ribotypes of toxigenic and non-toxigenic $C$. diphtheriae. C. diphtheriae exotoxin production depends on the presence of a lysogenic B-phage, which carries the gene encoding for the toxin. ${ }^{[4]}$

Humans are the only known reservoir for $C$. diphtheriae. It is spread by airborne respiratory droplets and direct contact with either respiratory secretions or infected skin lesion exudate. ${ }^{[4]}$

\section{Clinical manifestations of respiratory tract diphtheria}

C. diphtheriae infection occurs locally in the respiratory tract or skin due to non-invasive infection. Absorption and dissemination of diphtheria toxin result in bacteraemia, endocarditis and arthritis. The incubation period ranges from 2 to 4 days with local symptoms and signs of inflammation, described as follows: ${ }^{[4]}$

- Anterior nasal infection. Infection of the interior nares presents with a serosanguinous or seropurulent nasal discharge. There may be a whitish mucosal membrane present particularly on the septum. The discharge can incite an erosive reaction on the external nares and upper lip. Symptoms are usually mild. ${ }^{[4]}$

- Faucial infection. Pharyngeal fauces, the posterior mouth and proximal pharynx are the common sites for clinical diphtheria. Onset is over several days, with a low-grade fever, malaise and sore throat. Toxin elaboration locally induces a dense necrotic coagulum. Removal of this adherent grey-brown 'pseudomembane' reveals a bleeding, oedematous submucous. The underlying softtissue oedema and cervical adenitis can result in a characteristic bull-neck appearance, stridor and respiratory embarrassment. ${ }^{[4]}$
- Laryngeal and tracheobronchial infection. Laryngeal infection may begin de novo or spread from the pharynx. Presentation includes hoarseness, a brassy cough, stridor and dyspnoea. Oedema and membrane formation result in respiratory embarrassment, severe respiratory distress and cyanosis. Immediate membrane removal and intubation are required to prevent death. ${ }^{[4]}$

\section{Epidemiology of diphtheria}

Diphtheria is endemic in certain countries in Asia, Africa and South America. The World Health Organization (WHO) reported 7321 cases of diphtheria worldwide in 2014 (Table 1).

Table 1. WHO-reported cases of diphtheria in different regions of the world from 2010 to $2014 .{ }^{[5]}$

\begin{tabular}{llllll}
\hline & \multicolumn{5}{c}{ Reported diphtheria cases per annum, $\boldsymbol{n}$} \\
\cline { 2 - 6 } Region & $\mathbf{2 0 1 4}$ & $\mathbf{2 0 1 3}$ & $\mathbf{2 0 1 2}$ & $\mathbf{2 0 1 1}$ & $\mathbf{2 0 1 0}$ \\
\hline Africa & 1 & 128 & 27 & 13 & 50 \\
Americas & 8 & 5 & 2 & 12 & 57 \\
South-east Asia & 7217 & 4080 & 3953 & 5179 & 4120 \\
Europe & 33 & 33 & 32 & 33 & 39 \\
Eastern Mediterranean & 40 & 392 & 334 & 352 & 154 \\
Western Pacific & 22 & 42 & 142 & 37 & 153 \\
Global & 7321 & 4680 & 4490 & 5626 & 4573
\end{tabular}

In 1990, a major epidemic occurred in some of the countries of the former Soviet Union. Over 157000 cases and 5000 deaths were reported. ${ }^{[6]}$

In SA between January 2008 and March 2015, three laboratoryconfirmed cases of respiratory diphtheria were reported. Two of these were from Western Cape Province and one from Eastern Cape Province. These were followed by the KZN case in March 2015. ${ }^{[1]}$

\section{Case definition and classification: NICD May 2015}

Clinical case definitions

The clinical case definition for respiratory diphtheria is a person who presents with an upper-respiratory tract illness characterised by sore throat, low-grade fever and an adherent membrane of the nose, pharynx, tonsils or larynx.

Other presentations of diphtheria include patients presenting with:

- mild respiratory symptoms but no membrane,

- skin lesion, with C. diphtheria, C. ulcerans or C. pseudotuberculosis isolated from a nasopharyngeal swab or skin lesion swab, or

- rare presentations such as endocardial, laryngeal, conjunctival, otic or genital diphtheria.

Laboratory diagnostic criteria confirmation is by isolation of toxinproducing C. diphtheriae/C. ulcerans/C. pseudotuberculosis from a clinical specimen.

\section{Case classification}

- Suspected case: a person who meets the clinical case definition for respiratory diphtheria and has no laboratory confirmation and 
no epidemiological link to a laboratoryconfirmed case.

- Probable case: a person who meets the clinical case definition for respiratory diphtheria plus one of the following:

- Isolation of C. diphtheriae/C. ulcerans/ C. pseudotuberculosis but with toxigenicity status not yet confirmed

- An epidemiological link with a laboratory-confirmed case

or a person who presents with mild respiratory symptoms with no membrane or other presentations of diphtheria, but has an epidemiological link to a laboratory.

- Confirmed case: a person who meets the clinical case definition for respiratory diphtheria and is laboratory confirmed or a person presenting with mild respiratory symptoms with no membrane or other presentations of diphtheria and is laboratory confirmed.

- Symptomatic carrier: a person with no symptoms but for whom there is laboratory confirmation of a toxigenic strain.

- Discarded: a suspected or probable case in whom other compatible organisms are isolated or C. diphtheriae/ C. ulcerans/C. pseudotuberculosis is isolated but is confirmed to be a nontoxigenic strain.

In June 2015, a new category was added: ${ }^{[2]}$

- Possible case: ${ }^{[2]}$ a person who meets the clinical case definition for respiratory diphtheria and has no epidemiological link to a laboratory-confirmed case.

This definition accommodates a typical clinical presentation with a negative swab.

\section{Diphtheria treatment}

Diphtheria antitoxin

DAT is a hyperimmune antiserum produced in horses. DAT reduces mortality from $7 \%$ to $2.5 \%$. It is critical for the antibody to be administered as soon as a clinical diagnosis is made and before laboratory confirmation, as the antibodies will only neutralise the toxin before it enters the cell. The American Academy of Pediatrics recommends 20000 to 40000 units for pharyngeal or laryngeal disease of 48 hours' duration or less; 40000 to 60000 units for nasopharyngeal lesions; and 60000 to 120000 units for extensive disease of 3 or more days' duration or patients with brawny swelling of the neck. Hypersensitivity to horse serum must be assessed first. ${ }^{[4]}$

\section{Antibiotic therapy}

Penicillin and erythromycin are generally recommended. By killing the organism, the antibiotic terminates toxin production, ameliorates local infection and prevents spread to uninfected contacts. ${ }^{[4]}$

\section{Diphtheria vaccination in SA}

Diphtheria toxoid was developed in 1921 and has been used routinely since 1940. Diphtheria toxoid is combined with tetanus toxoid (DT) as a paediatric or adult formulation (Td) and with acellular pertussis vaccine as DTaP and TDap. The paediatric formulation contains three to four times as much diphtheria toxoid as the adult dose.$^{[8]}$ Diphtheria serum concentrations of $0.01-0.1 \mathrm{IU} / \mathrm{mL}$ are thought to confer protection. ${ }^{[4]}$ Data from one outbreak showed that $90 \%$ of clinical cases had antitoxin levels below $0.01 \mathrm{IU} / \mathrm{mL} .{ }^{[4]}$ In the same outbreak, 92\% of asymptomatic carriers had concentrations above $0.1 \mathrm{IU} / \mathrm{mL}^{[4]}$ After immunisation, antitoxin levels decline slowly, so that up to $50 \%$ of adults over 60 years of age have serum titres below $0.01 \mathrm{IU} / \mathrm{mL}$. Therefore, booster doses are recommended every 10 years. ${ }^{[4]}$

\section{Postulates for the decreasing incidence of diphtheria in the West}

A number of postulates have been put forward to explain the decreasing incidence of diphtheria in the West:

- Historical evidence suggests diphtheria occurs in cycles of 100 years or more.

- Organisms isolated from immunised individuals are less likely to be toxigenic than from those who are unimmunised.

- Local elaboration of toxin, in the absence of antibody, enhances an organism's ability to colonise, and immunisation with toxoid could counteract this selective advantage of toxigenic strains.

- Virulence factors other than toxin production may exist.

- Protection may correlate with lower serum concentrations of antitoxin levels or immune mechanisms that are untested. ${ }^{[4]}$

In the diphtheria epidemic in the former Soviet Union, $50 \%$ of cases occurred in individuals aged 15 years or older. This suggested that the young were protected by infant immunisation and older people were vulnerable because of lack of childhood immunisation or fading antibody levels. ${ }^{[2]}$ Good paediatric immunisation schedules appear to be sufficient for keeping toxigenic strains from circulation and causing adult disease. Efficient immunisation programmes require $90 \%$ coverage to remain effective. ${ }^{[4]}$

The SA paediatric immunisation programme provides DTaP in a hexavalent combination vaccine at 6,10 and 14 weeks of age and at 18 months of age. Two booster doses of TD are given at 6 and 12 years of age. In addition, $\mathrm{Td}$ vaccine is administered to individuals who have had tetanus-prone injuries.

WHO and the United Nations Children's Fund (UNICEF) estimated national immunisation coverage in SA in 2014 to be approximately $80 \%$ for DTP1 and $70 \%$ for $\mathrm{DTP}^{\left[{ }^{[9]}\right.}$ (Figs 2 and 3).

SA has ensured universal access to vaccines, and rapid introduction of new vaccines into the national Expanded Programme of Immunisation; however, without highquality surveys, the uncertainty of vaccine coverage in SA remains a challenge. ${ }^{[10]}$

\section{Effect of immigrants and refugees on an immunisation programme}

South Africa (SA) offers free routine childhood immunisations to all children irrespective of nationality. SA border controls check for all routine vaccinations in individuals entering the country but not routine childhood vaccination. Immigrants may be uneducated about immunisation, have preconceived ideas about it or be unaware of local protocols.

An Australian study conducted in 2011 reported that immigrant children from east Africa are likely to be inadequately immunised. Patient recall was also unreliable, as it did not correlate with serum antibody levels. ${ }^{[11]}$ Another immigrant study in Minnesota, USA found that $81.1 \%$ of refugees lacked the necessary documentation for having received the three doses of diphtheria and tetanus vaccines. Documentation rates were lowest for refugees from sub-Saharan Africa. ${ }^{[12]}$

A study carried out in Israel analysed immigrants from the former Soviet Union. Of males between 17 and 19 years of age, 


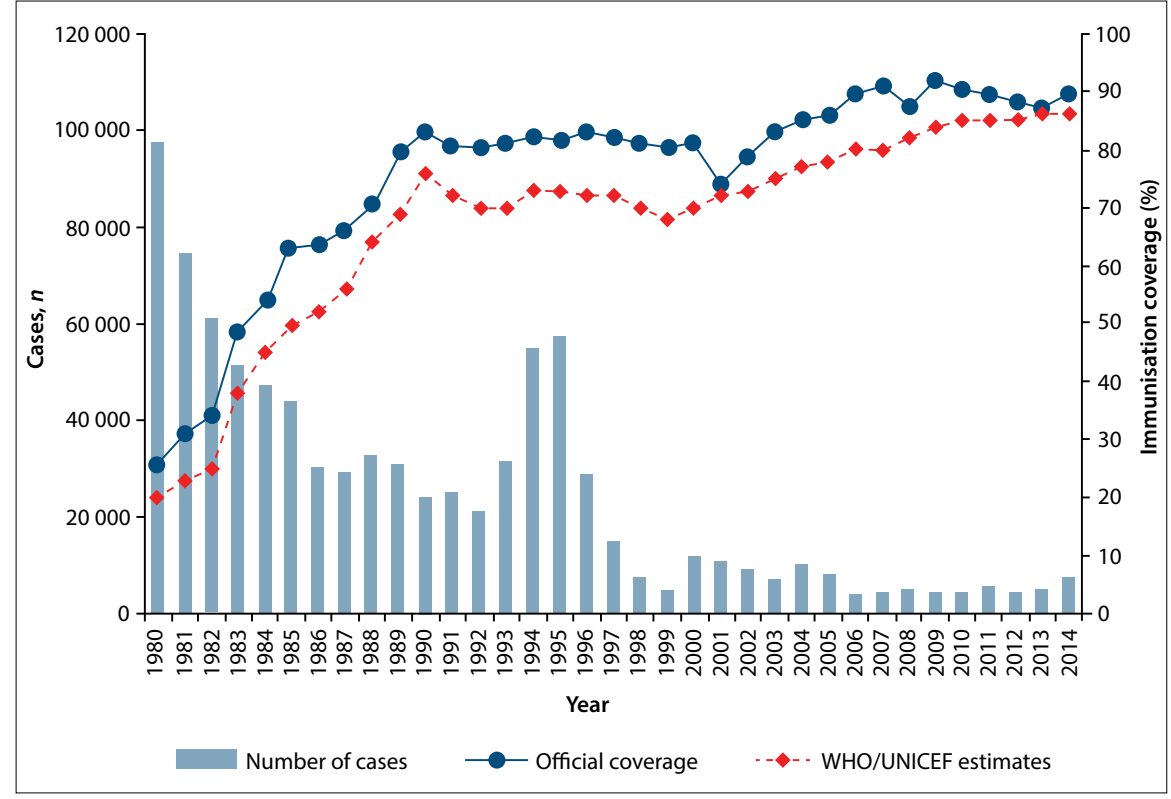

Fig. 2. Global annual reported cases of DTP3 coverage between 1980 and 2014. ${ }^{[5]}$

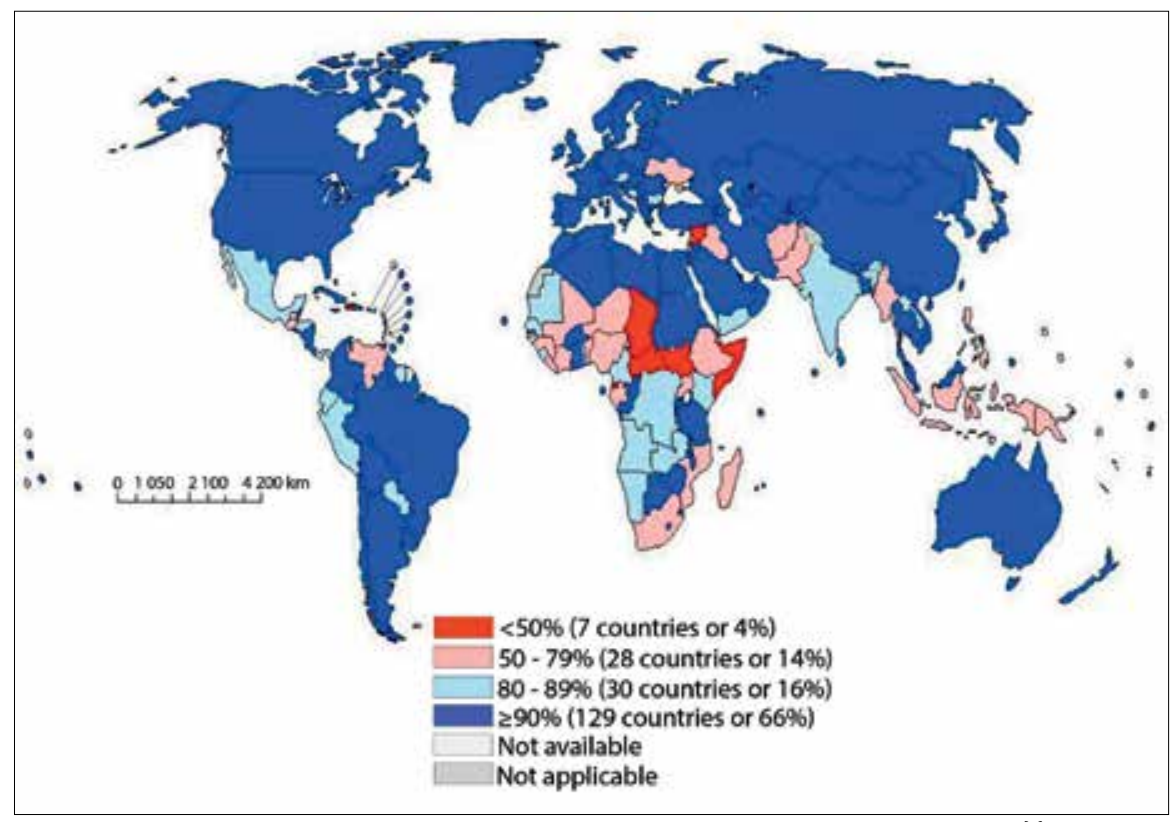

Fig. 3. World map of immunisation coverage with DTP3 vaccine in infants in 2014. ${ }^{[5]}$

$4.8 \%$ had antitoxin antibody levels below the protective level of $0.01 \mathrm{IU} / \mathrm{mL}$. The research also found that the immigrants appeared more susceptible to diphtheria, and recommended they receive booster doses of diphtheria toxoid. ${ }^{[13]}$ However, an analysis in Greece of eastern European immigrants' diphtheria status actually found lower immunity rates among Greek adults. It was suggested that Greek adults had no natural contact with toxigenic strains of Corynebacteria and recommended they receive booster doses, to reduce their risk of acquiring toxigenic strains from individuals who may carry the of factors: there were many unnecessary contraindications to vaccination; the breakup of the Soviet Union led to large-scale population movements; and there was lack of adequate supplies and disruption to health services. ${ }^{[9]}$ Following that outbreak, a number of identified strategies were implemented to decrease the incidence of diphtheria. These included the following:

- The introduction by some European countries of adult booster vaccination every 10 years, or as part of a programme to administer $\mathrm{Td}$ vaccine for tetanusprone injuries ${ }^{[9]}$

- The introduction of adult vaccination surveys by the Latvian government ${ }^{[9]}$

- Greater attention given to checking the vaccination records of high-risk groups. In Latvia, new recruits into the military were given the diphtheria vaccine where appropriate. ${ }^{[9]}$

Post-Dominican Republic epidemic

In 2004 - 2005, the largest diphtheria outbreak this century in the western hemisphere occurred in the Dominican Republic and Haiti. ${ }^{[15]}$ In contrast to the outbreak in the former Soviet Union, children aged 1 - 4 years were the most at risk. Children living in low-income urban areas with difficult access to vaccination were affected. The main factor in the outbreak was a low vaccine coverage rate $(<90 \%)$. The outbreak resulted in revision of the diphtheria case definition and epidemiology case investigation procedure. Protocols for clinical management protocols and laboratory specimen collection were updated and widely distributed, with laboratory diagnostic capabilities improved. Management of diphtheria and access to the DAT was improved. ${ }^{[15]}$

\section{Insights from the KZN diphtheria outbreak}

SA should consider developing its policies and programmes to offer immunisation to all immigrants. Healthcare providers should be vigilant in providing immunisations when these children do present to healthcare facilities. The introduction of an adult booster vaccine every 10 years is also suggested.

\section{International post- epidemic surveys}

Post-European epidemic

Resurgence of diphtheria in Europe in 1990 was thought to be caused by a number
Following the KZN diphtheria outbreak, a number of areas of vulnerability to future outbreaks were identified:

- Power outages due to 'load-shedding' of electricity have resulted in an unreliable vaccine cold chain.

- SA has limited financial capacity for vaccine surveillance.

- SA continues to have $<90 \%$ vaccine coverage.

- The presence of illegal and legal immigrant and refugee population 
groups poses a risk of introducing pockets of unvaccinated individuals.

- Children who do not attend school or clinics may miss out on routine and catch-up immunisation programmes.

- Poor maternal education and lack of access to healthcare because of financial difficulty or residence in remote areas promotes noncompliance with the local vaccine schedule.

- Diphtheria is not endemic in SA, so a stock of the DAT is not maintained.

- Diphtheria booster vaccines are not offered to adults routinely every 10 years.

The SA response to the epidemic was as follows:

- The NICD case definition was updated.

- The SA diphtheria guidelines and laboratory guidelines were updated and widely distributed.

- Vaccine campaigns were implemented in KZN to administer booster vaccine and catch-up immunisations.

- Antimicrobials were provided to contacts.

- The epidemic was widely publicised in the media to increase public awareness.

- The KZN Department of Health, University of KZN, NICD and a consultant from the $\mathrm{WHO}$ are reviewing the epidemic to determine appropriate future interventions.

- Emergency access to DAT was obtained by humanitarian assistance from Japan.

- The KZN Department of Health led widespread education programmes.

- The NICD has requested that all laboratories submit stored or prospectively identified Corynebacterium isolates to them for molecular characterisation. ${ }^{[3]}$

In summary, the KZN diphtheria outbreak has highlighted factors that make SA vulnerable to disease outbreaks, but has also shown that collaborative effort and far-sightedness may be able to prevent or limit future outbreaks. SA needs to maintain a robust immunisation programme with high-level surveillance programmes.

\section{References}

1. National Institute of Communicable Diseases. Communicable Diseases Communiqué 2015;14(3). http://www.nicd.ac.za/assets/files/NICD-NHLS (accessed 22 February 2016).

2. National Institute of Communicable Diseases. Communicable Diseases Communiqué 2015;14(6). http://www.nicd.ac.za/assets/files/NICD-NHLS (accessed 22 February 2016).

3. National Institute of Communicable Diseases. Communicable Diseases Communiqué 2015;14(8). http://www.nicd.ac.za/assets/files/NICD-NHLS (accessed 22 February 2016).

4. MacGregor R, Bennett JE. Corynebacterium diphtheria. In: Dolin R, Blaser MJ (eds). Mandell, Douglas, and Bennett's Principles and Practice of Infectious Diseases, 8th ed. Philadelphia: Elsevier; 2015:2366-2372.

5. World Health Organization. Global Health Observatory Data Repository. Geneva: WHO, 2015. http://apps.who.int/gho/data/view.main (accessed 2 April 2016).

6. Wagner KS, White JM, Lucenko I, Mercer D, Crowcraft NS, et al. Diphtheria in the post-epidemic period, Europe 2000 - 2009. Emerg Infect Dis J 2012;18(2):217-225. DOI:10.3201/eid1802.110987

7. National Institute of Communicable Diseases. Recommendations for the Management and Public Health Response to Diphtheria, Version 1. Johannesburg: Division of Public Health Surveillance and Response, NICD, National Health Laboratory Service, 2015.

8. Centers for Disease Control and Prevention. Epidemiology and Prevention of VaccinePreventable Diseases - The Pink Book. 13th ed. Atlanta, Georgia: CDC, 2015:107-118.

9. World Health Organization and United Nations Children's Emergency Fund. Estimates of National Immunisation Coverage. Geneva: WHO, 2015. http://apps. who.int/gho/data/view.main (accessed 2 April 2016).

10. Madhi SA, Bamford L, Ngcobo N. Effectiveness of pneumococcal conjugate vaccine and rotavirus introduction into the SA public immunisation programme. S Afr Med J 2014;104(3Suppl1):228-234. DOI:10.7196/samj.7597

11. Paxton GA, Rice J, Davie G, Carapetis JR, Skull SA. East African immigrant children in Australia have poor immunisation coverage. J Paediatr Child Health 2011;47(12):888892. DOI:10.1111/j.1440-1754.2011.02099.x

12. Lifson $A R$, Thai $D$, Hang K. Lack of immunization documentation in Minnesota refugees: Challenges for refugee preventative health care. J Immigr Health 2001;3(1):47-52.

13. Low M, Almog R, Green MS, et al. Immune status against diphtheria among immigrants from the former USSR who arrived in Israel during 1990 - 1991. Infection 1998;26(2);104-108. DOI:10.1007/bf02767769

14. Pournaras S, Tsakris A, Hadjichristodoulou C, et al. Diphtheria immunity of Albanian and other eastern European immigrants in Greece compared with the local population - the risk of re-emergence in Greece. Infection 1999;27(6):361-364. DOI:10.1007/s150100050044

15. Garib Z, Danovaro-Holliday CM, Tavarez Y, Leal I, Pedreira C. Diphtheria in the Dominican Republic: Reduction of cases following a large outbreak. Pan Am J Pub Health 2015;38(4):292-299. 